\title{
Trombocitopenia inmune primaria. Tratamiento y recomendaciones ante la pandemia por COVID-19
}

\section{Gregorio Campos-Cabrera1', Monica Lozano-Garcidueñas² y Carlos Martínez-Murillo3*}

${ }^{1}$ Departamento de Hematología y Trasplante, Laboratorios Fátima de Michoacán. Morelia, Mich.; ${ }^{2}$ Departamento de Hematología Pediátrica, Hospital GEneral de Zona N. ${ }^{0}$ 14, IMSS, Hermosillo, Son.; ${ }^{3}$ Departamento de Hematología, Unidad 111D, Hospital General de México Eduardo Liceaga, Ciudad de México. México

\section{Resumen}

La trombocitopenia inmune primaria (TIP) es una trastorno autoinmune adquirido caracterizado por el recuento bajo de plaquetas como resultado de la destrucción de plaquetas y la producción de plaquetas deteriorada. La falta de ensayos aleatorizados sobre el manejo de la TIP ha dado como resultado controversias significativas y gran variación en la práctica clínica para su diagnóstico y tratamiento. Aunado a esto, la pandemia actual de enfermedad por coronavirus 2019 (COVID-19), causada por el nuevo coronavirus 2 del síndrome respiratorio agudo grave (SARS-CoV-2), plantea una serie de dilemas para el estudio y tratamiento de los pacientes con trombocitopenia inmune, incluyendo las ventajas y desventajas de las opciones terapéuticas estándar para la TIP de reciente diagnóstico o en recaída y cronicidad, en cada uno de los enfoques de manejo: observación, corticosteroides, inmunoglobulina intravenosa, inmunoglobulina anti-D (anti-Dlg), rituximab, esplenectomía y agonistas de los receptores de trombopoyetina, así como el reconocimiento de los desafíos planteados al manejo de pacientes con TIP con manifestaciones hemorrágicas y riesgo trombótico observado en pacientes hospitalizados con infección por SARS-CoV-2. Por tal motivo, el presente trabajo tiene como objetivo plasmar y ofrecer recomendaciones y pautas a seguir tanto en niños como en adultos con y sin COVID-19, ante el riesgo y beneficio en cada escenario, basado en consenso de expertos y guías ya establecidas (sobre todo para pacientes adultos) para el manejo, tratamiento y seguimiento de la TIP en el contexto de la actual pandemia.

PALABRAS CLAVE: Trombocitopenia inmune. COVID-19. Pandemia. Tratamiento. Riesgos.

\section{Primary immune thrombocytopenia. Treatment and recommendations for the COVID-19 pandemic}

\begin{abstract}
Primary immune thrombocytopenia (ITP) is an acquired autoimmune disorder characterized by low platelet count as a result of platelet destruction and impaired platelet production. The lack of randomized trials on the management of IPT has resulted in significant controversy and great variation in clinical practice for its diagnosis and treatment. In addition to this, the current coronavirus disease 2019 (COVID-19) pandemic, caused by the new severe acute respiratory syndrome coronavirus 2 (SARS-CoV-2), poses a series of dilemmas for the study and treatment of patients with immune thrombocytopenia, including the advantages and disadvantages of therapeutic options standard for newly diagnosed or relapsed and chronic IPT, in each of the management approaches: observation, corticosteroids, IV immunoglobulin, anti-D immunoglobulin, rituximab, splenectomy and thrombopoietin receptor agonists, as well as recognition of the challenges posed to the management of patients with IPT with hemorrhagic manifestations and thrombotic risk observed in hospitalized patients with SARS-CoV-2 infection. For this reason, the present work aims to capture and offer recommendations and guidelines to follow in both children and adults with


and without COVID-19, given the risk and benefit in each scenario, based on consensus of experts and already established guidelines (on all for adult patients) for the management, treatment and follow-up of IPT in the context of the current pandemic.

KEYWORDS: Immune thrombocytopenia. COVID-19. Pandemic. Treatment. Risks.

\section{Introducción}

La trombocitopenia inmune ( $\mathrm{TI})$ es un síndrome autoinmune que involucra la destrucción de plaquetas mediada por anticuerpos y linfocitos autorreactivos acompañada de una disminución en la producción de plaquetas y que puede predisponer a sangrados. El consenso internacional para la definición, tratamiento y criterios de respuesta recomiendan que el término TI sea utilizado para designar todos los tipo de trombocitopenia autoinmune; si existe una causa clínica subyacente o exposición a medicamentos será TI secundaria, de lo contrario será TI primaria (TIP) y su diagnóstico es de exclusión, además el recuento plaquetario en ambos casos debe ser menor a $100 \mathrm{x}$ $10 \%$. Recientemente se ha sugerido que para considerar TI el paciente debe de tener un recuento plaquetario menor a $20 \times 10^{9} / \mathrm{l}$ y responder a esteroides, inmunoglobulina intravenosa (IgIV) o tratamiento de la causa secundaria con incremento a cuando menos $30 \times 10^{9} / /$ plaquetas $^{1-5}$.

Se estima una incidencia de TIP de 2 a 5 por cada 100,000 personas en la población general. Es importante clasificar a la TIP de acuerdo con el tiempo de evolución, ya que de esto dependerán las recomendaciones de tratamiento. Durante los primeros 3 meses al diagnóstico se considera TIP aguda, de 3 a 12 meses TIP persistente y mayor a 12 meses, TIP crónica $^{2-3}$.

Su fisiopatología depende de varios mecanismos. No se ha encontrado el evento inicial que origina este síndrome autoinmune, pero se ha demostrado susceptibilidad genética e infecciones como desencadenantes. Los autoanticuerpos contra las glucoproteínas plaquetarias son la característica principal de la fisiopatología, se encuentran presentes en la mayoría de los pacientes, favorecen la depuración de plaquetas a través del sistema retículo endotelial mediante sus receptores $\mathrm{Fc}$, principalmente en bazo. Las plaquetas y los megacariocitos comparten la presencia de glucoproteínas, por lo que los anticuerpos inducen una proliferación y diferenciación defectuosa de megacariocitos, y alteran la producción de proplaquetas. Los niveles séricos de trombopoyetina se encuentran disminuidos en relación con la cuenta plaquetaria debido al incremento en la masa total de megacariocitos y la depuración acelerada de plaquetas. El daño tóxico directo de linfocitos $T$ autorreactivos hacia las plaquetas y megacariocitos ha sido bien documentado, en paciente con y sin autoanticuerpos circulantes. Recientemente se ha estudiado el sistema del receptor Ashwell-Morrell en la depuración de plaquetas al disminuir la cantidad de ácido siálico durante su senescencia y la producción de trombopoyetina ${ }^{6-9}$.

El tratamiento estándar de la TIP en sus diversas etapas de evolución, aguda, persistente y crónica, ya se encuentra recomendado por guías nacionales e internacionales ${ }^{5,10-12}$. Ante la pandemia por COVID-19 el tratamiento estándar no ha sido bien establecido, por lo que deben ser diseñadas recomendaciones para que sirvan de apoyo a los médicos en el tratamiento de TIP durante esta pandemia. Hasta junio de 2020 solo se habían publicado las guías del UK ITP Forum $^{13}$. Las recomendaciones de la American Society of Hematology (ASH) se mencionan en su página de apoyo, basadas en consenso de expertos ${ }^{14}$. Para el abordaje de las recomendaciones, plantearemos los siguientes escenarios clínicos: a) trombocitopenia y COVID-19, b) manejo en TIP aguda y en recaída, c) manejo en TIP persistente y crónica, d) riesgo trombótico en TIP, y e) TIP en niños.

\section{Trombocitopenia y COVID-19}

La trombocitopenia en pacientes infectados por SARS-CoV-2 es generalmente leve, cuentas por debajo de 100,000 se encuentran en el $5 \%$ de los hospitalizados en sala general y en el $8 \%$ de los hospitalizados en terapia intensiva ${ }^{15,16}$. La fisiopatología es multifactorial y más compleja que el convencional modelo de consumo, incluyendo la marcada activación plaquetaria mediada por trombina, el uso de antibióticos, antivirales y heparinas, entre otros medicamentos y procedimientos como hemodiálisis y oxigenación por membrana extracorpórea, que pueden contribuir $^{13}$. El grado de trombocitopenia está relacionado directamente con la gravedad de la enfermedad y es un factor pronóstico adverso y fatal; se ha reportado que en los pacientes fallecidos la trombocitopenia se presentó en el $20 \%$ de ellos en comparación 
con el $1 \%$ de los sobrevivientes $(p<0.0001)^{17,18}$. El diagnostico de TIP es por exclusión, por lo que deben descartarse otras etiología. Para sospechar causa inmunitaria se debe tener un descenso súbito de plaquetas a menos de $20 \times 10 \%$ o un descenso del $50 \%$ en un periodo de 24 a 48 horas. Dentro de las causas de tipo inmunitario que considerar están la púrpura trombocitopénica trombótica y el síndrome urémico hemolítico atípico, que deben ser considerados ante la presencia de anemia hemolítica microangiopática y niveles de ADAMTS-13 menores al 10\% de actividad. La TI inducida por heparina se presenta entre 5 a 10 días de la primera exposición 0 a las 24 h en exposiciones posteriores. Al igual que la trombocitopenia inducida por medicamentos, que se presenta en promedio a los 14 días de la primera exposición a un nuevo medicamento o antes si es exposición subsecuente ${ }^{13}$.

\section{Puntos clave y recomendaciones}

- La trombocitopenia grave es poco frecuente en la infección por SARS-CoV-2, se presenta por lo general hasta la etapa terminal.

- Recuento de plaquetas $<20 \times 10^{9} /$ o descenso súbito de $>50 \%$ en un periodo de 24 a 48 horas sugieren causa inmunitaria.

- Se deben excluir otras etiologías antes de diagnosticar TIP.

\section{Manejo en trombocitopenia inmune primaria aguda y en recaída}

La COVID-19 puede condicionar TIP de reciente diagnóstico o recaída en paciente con diagnóstico ya establecido, como ya ha sido ampliamente reportado $^{19-23}$. Sin embargo, son reportes de casos y el tratamiento ha sido con base en las características individuales de cada paciente. La decisión sobre los medicamentos que utilizar estará determinada por si el paciente es COVID-19 negativo o COVID-19 positivo:

- Paciente COVID-19 negativo. El tratamiento de primera línea en pacientes con TIP aguda y en recaída sin COVID-19 puede ser con esteroides, como lo indican las guías actuales ${ }^{5,10-12}$. Ya que no existen datos suficientes que sugieran que los esteroides incrementan el riesgo de desarrollar infección por SARS-CoV-2 o que esta tenga una evolución adversa. La Organización Mundial de la Salud (OMS) ha recomendado limitar el uso de esteroides para el tratamiento de diversas enfermedades si existen otras opciones ${ }^{24}$, por lo que el uso de agonistas de los receptores de trombopoyetina (AR-tpo) e inmunoglobulina $G$ intravenosa (IgG IV), solos o en combinación, puede ser una alternativa razonable, ya que ninguno de los dos es inmunosupresor.

- Paciente COVID-19 positivo. El tratamiento de primera línea en pacientes con TIP aguda y en recaída con COVID-19 es con esteroides ${ }^{13,14}$. Se recomiendan las dosis más bajas y el menor tiempo posibles, $20 \mathrm{mg}$ de prednisona diario independientemente del peso del paciente durante dos semanas y posteriormente dosis de reducción, ya que pueden retardar la eliminación del coronavirus en los tejidos de los pacientes ${ }^{25}$. La IgG IV puede utilizarse de primera línea sola o en combinación con esteroides ${ }^{13,14}$, ya que produce un incremento rápido del conteo de plaquetas y ahorra la intensidad y duración de la terapia con esteroides. Aunque la OMS sugiera limitar el uso de esteroides, otras alternativas terapéuticas tienen mayor riesgo de incrementar la severidad de la infección por SARS-CoV-2. Los AR-tpo se han asociado a un mayor riesgo de eventos tromboembólicos y al desarrollo de micropartículas generadoras de trombina a las dos semanas de haber iniciado el tratamiento ${ }^{26}$, lo que pudiera contribuir a una mayor severidad de la coagulopatía por COVID-19. El uso de rituximab y otros inmunosupresores debe evitarse en lo mayor posible, ya que producen una depleción de linfocitos B por largo tiempo y por lo tanto se inhibe la formación de anticuerpos, lo que conduciría a una mayor gravedad de la infección por SARS-CoV-2 y por oportunistas ${ }^{13,14}$. La transfusión de plaquetas no se recomienda de forma sistemática, ya que puede incrementar el riesgo de eventos trombóticos; su uso debe limitarse a los casos con sangrado que pone en riesgo la vida o la función.

\section{Puntos clave y recomendaciones}

- En pacientes que son negativos para SARS-CoV-2, los AR-tpo puede preferirse como tratamiento de primera línea para evitar los corticosteroides, que pueden aumentar el riesgo de infección por SARS-CoV-2 durante la pandemia.

- En pacientes que son positivos para SARS-CoV-2: 
- Los AR-tpo deben evitarse (potencial aumento de riesgo trombótico).

- Si se usan esteroides como terapia de primera línea, la dosis y la duración debe mantenerse al mínimo necesario: dosis inicial de $20 \mathrm{mg}$ diarios en pacientes sin hemorragia, con aumento a $1 \mathrm{mg} / \mathrm{kg}$ después $3-5$ días si no ha habido respuesta y posterior esquema de reducción después de 2 semanas (lentamente si ha habido una buena respuesta, rápidamente si no hay respuesta).

- IgIV (1 g/kg) como primera línea si se requiere elevación inmediata del recuento de plaquetas por hemorragia.

- También se puede usar como tratamiento de segunda línea si no hay respuesta a esteroide.

- Las transfusiones de plaquetas solo deben administrarse si hay hemorragia que pone en riesgo la vida.

\section{Manejo en trombocitopenia inmune primaria persistente y crónica}

La reciente actualización 2019 de las guías de la ASH en adultos establece:

- En adultos con TPI que dura $\geq 3$ meses que dependen de corticosteroides o no responden a los corticosteroides, el panel de guías de la ASH sugiere:

- Esplenectomía o AR-tpo.

- Rituximab en lugar de esplenectomía.

- AR-tpo en lugar de rituximab.

- En adultos con TPI persistente o crónica después del cese de esteroides, el informe de consenso internacional recomienda terapia médica (ARtpo, rituximab, fostamatinib) durante 12-24 meses antes de considerar la esplenectomía ${ }^{5}$.

Los pacientes con TIP persistente y crónica estables deben de permanecer con su tratamiento sin modificación por la COVID-19. Esto es para todos los medicamentos, incluyendo esteroides, AR-tpo e inmunosupresores. Sin embargo, habrá que considerar omitir el uso de rituximab por la depleción de linfocitos B a largo plazo, lo que ocasionaría una inmunidad humoral deficiente en caso de infección por SARSCoV-2, menor eliminación de la carga viral y, por lo tanto, mayor riesgo de enfermedad grave. Los pacientes con esplenectomía no se encuentran en mayor riesgo de COVID-19, pero sí por agentes bacterianos, por lo que deben cumplir esquemas de antibióticos profilácticos y esquema de vacunación. En los casos anteriores el aislamiento protector y las medidas generales deben de observarse rigurosamente. Los pacientes que no hayan recibido tratamiento durante los últimos 12 meses deberán seguir las medidas generales de la población sin TIP ${ }^{13,14}$.

\section{Puntos clave y recomendaciones}

- Los pacientes con TIP crónica estables deben continuar con su medicación actual, pero con aislamiento y medidas preventivas rigurosas.

- Los pacientes con esplenectomía deben mantener cumplimento estricto de antibióticos profilácticos, inmunizaciones y estrictas medidas generales de prevención de infecciones.

- Los pacientes en vigilancia, apego a medidas generales de la población sin TIP.

\section{Riesgo trombótico en trombocitopenia inmune primaria}

Los pacientes con TIP se encuentran en mayor riesgo de eventos trombóticos venosos y arteriales que la población general, tanto por la fisiopatología de la enfermedad como por el tratamiento, principalmente con esplenectomía y con AR-tpo ${ }^{27}$. Los pacientes con infección por SARS-CoV-2 cuando son hospitalizados también tienen mayor riesgo de eventos trombóticos venosos y arteriales que la población general y existes algoritmos de profilaxis y tratamiento estandarizados en pacientes COVID-19 positivos ${ }^{28}$ : a) profilaxis antitrombótica con heparinas de bajo peso molecular (HBPM) en pacientes hospitalizados, b) dosis de HBPM basada en evidencia clínica e incremento de dosis en pacientes con enfermedad grave, c) uso selectivo de profilaxis posterior al egreso hospitalario de acuerdo con escalas de riesgo, y d) anticoagulación por mínimo 3 meses en pacientes que desarrollaron un evento trombótico.

La HBPM/heparina se usa ampliamente como tromboprofilaxis en todos los pacientes hospitalizados con COVID-19 y debe administrarse incluso a pacientes con TIP. Los beneficios potenciales frente al riesgo de HBPM/heparina y su mejor dosis y horario deben sopesarse cuidadosamente en cada paciente con TPI individualmente, dada la escasez de datos sobre hipercoagulabilidad en pacientes con TPI y COVID-19.

En caso de que el paciente necesite tratamiento anticoagulante profiláctico o a dosis plenas, habrá que considerar si cursa o no con trombocitopenia. Si 
no hay trombocitopenia las dosis habituales para profilaxis y anticoagulación formal deben de seguirse de acuerdo con las guías ${ }^{28}$. De existir trombocitopenia, esas mismas guías deben considerarse, así como las recomendaciones específicas de pacientes con TIP que requieren anticoagulación ${ }^{29,30}$ : anticoagulación profiláctica con plaquetas por arriba de $30 \mathrm{x}$ $10 \%$, por debajo de esta cifra considerar compresión neumática intermitente; anticoagulación formal con plaquetas por arriba de $50 \times 10^{\%} / \mathrm{y}$ con reducción de la anticoagulación formal en un $50 \%$ con plaquetas entre 30 y $50 \times 10^{9} / /^{13,27-30}$.

\section{Puntos clave y recomendaciones}

- Los pacientes con TIP y COVID-19 positivos que se encuentre hospitalizados deben recibir profilaxis antitrombótica de acuerdo con el peso del paciente cuando las plaquetas se encuentren por arriba de $30 \times 10^{9} /$ y no exista contraindicación o hemorragia; compresión neumática intermitente cuando estén por debajo de esa cifra.

- En los pacientes con TIP y COVID-19 positivos que necesiten anticoagulación, se recomienda dosis completa con plaquetas por arriba de $50 \mathrm{x}$ $10^{9} /$ y reducción de la dosis en un $50 \%$ con plaquetas entre 30 y $50 \times 10 \%$; con cifras menores a $30 \times 10 \%$ considerar otras alternativas no farmacológicas.

- Valorar constantemente el riesgo trombótico y hemorrágico para hacer los ajustes necesarios.

\section{Trombocitopenia inmune primaria en el paciente pediátrico}

La TIP es la enfermedad adquirida hemorrágica más común en la infancia. La respuesta autoinmune frecuentemente es desencadenada por infecciones virales, como citomegalovirus, virus de Epstein-Barr, parvovirus B19, rubeola y sarampión; también por inmunizaciones y tratamiento con antivirales; y tiene una evolución autolimitada en la mayoría de los $\operatorname{casos}^{31,32}$.

A pesar de un aumento en el número de terapias disponibles para tratar a pacientes con TIP, hay datos mínimos de ensayos aleatorizados que soporten el manejo de pacientes. Por lo que la ASH estableció pautas basadas en evidencia, con el objetivo de apoyar en la toma de decisiones en TIP.

En la actualización 2019 de las guías ASH para la TIP en niños, destacan algunas diferencias entre las guías previas (2011): a) se recomienda manejo ambulatorio en pacientes sin o mínima manifestación hemorrágica, b) los corticosteroides se recomiendan sobre IgG IV o anti-D, consideradas también de primera línea, c) se establece el tipo de esteroide y el régimen de este, se recomienda curso corto de prednisona, y d) se prioriza evitar la hospitalización, se prioriza la observación y considerar una amplia variedad de factores para la toma de decisión terapéutica correcta e individualizada.

Se establece en el tratamiento de primera línea para los pacientes pediátricos como sigue:

- En niños con TIP de reciente diagnóstico sin manifestación de sangrado o solo sangrado leve o menor, independiente del recuento de plaquetas (mayor o menor de $20 \times 10 \%$ /) se recomienda:

- Observación en lugar de corticosteroides.

- Observación en lugar de la IgIV.

- Observación en lugar de anti-Dlg.

- En niños con TIP de reciente diagnóstico con manifestación de sangrado que no pone en riesgo la vida y/o disminuye la calidad de vida por riesgo de sangrado, el panel sugiere:

- Corticosteroides más que anti-Dlg.

- Corticosteroides más que IgIV.

- Anti-Dlg mas que IgIV.

- En niños con TIP de reciente diagnóstico con sangrado mucoso sin riesgo para la vida y/o disminución de la calidad de vida por riesgo de sangrado, en el panel de la ASH se declara:

- En contra de cursos de corticosteroides mayores a 7 días; a favor de cursos de 7 días 0 menos.

- La dosis recomendada de corticosteroide se sugiere como sigue: prednisona, 2-4 mg/kg/día, máximo $120 \mathrm{mg}$ diarios, durante 5-7 días; en lugar de dexametasona $0.6 \mathrm{mg} / \mathrm{kg} / \mathrm{día}$, máximo $40 \mathrm{mg}$ por día durante 4 días.

En el tratamiento de segunda línea, en pacientes pediátricos con TIP de más de 3 meses (persistente o crónica), el panel de expertos consideró a los agentes agonistas de trombopoyetina, rituximab y esplenectomía, debiendo considerar para la elección de estos la edad, la actividad, el riesgo de sangrado, la duración de la TIP, si es primaria o secundaria, los tratamientos previos e incluso la preferencia del paciente, priorizando evitar inmunosupresión y tratamientos a largo plazo, con decisión del tratamiento individualizada ${ }^{5}$.

- En niños con TIP de reciente diagnóstico con sangrado mucoso sin riesgo para la vida $y / 0$ 
disminución de la calidad de vida por riesgo de sangrado, y que no responden al tratamiento de primera línea, la ASH sugiere:

- AR-tpo en lugar de rituximab.

- AR-tpo en lugar de esplenectomía.

- Rituximab en lugar de esplenectomía.

La COVID-19 en la mayoría de los niños tiene un curso generalmente asintomático o con manifestaciones clínicas leves, con presentación de una enfermedad leve a moderada de corta duración y recuperación acelerada y buen pronóstico en comparación con los adultos, siendo esporádicos los casos severos o graves y con una nula tasa de mortalidad. Pocos pueden evolucionar a infección de las vías respiratorias bajas. Por otra parte, se ha descrito recientemente la participación gastrointestinal, manifestaciones dérmicas y manifestación de un síndrome similar a las manifestaciones de la enfermedad de Kawasaki. La trombocitopenia no se encuentra como característica en las alteraciones de laboratorio ${ }^{33-37}$.

Hasta la fecha, aún no se ha descrito la existencia de una asociación entre TIP y la COVID-19, así como si el impacto en el recuento de plaquetas difiere de aquel en infecciones con otros virus conocidos. Sin embargo, los niños afectados por la TPI podrían ser más susceptibles a enfermedades infecciosas que otras en general. En niños inmunocomprometidos las infecciones por otros coronavirus causan manifestaciones más severas que en los niños inmunocompetentes ${ }^{38}$, definiendo como inmunocomprometidos a los niños con inmunodeficiencias primarias, que reciben quimioterapia para neoplasia hematológica 0 sólida, tratamiento inmunosupresor crónico, trasplante hematopoyético y de otros órganos.

Actualmente se desconoce si un estado de inmunosupresión puede contribuir a empeorar el resultado en niños durante la infección por SARS-CoV- $2^{37}$. Sin embargo, es ampliamente reconocido que la TIP puede ser una posible manifestación secundaria de otros trastornos hematológicos e inmunitarios caracterizados por deficiencia del sistema inmunitario celular y/o humoral, como la inmunodeficiencia variable común, deficiencia selectiva de IgA y síndrome de DiGeorge. Además, el uso de algunos medicamentos podría exponer a los pacientes con TIP a riesgos adicionales en comparación a la población general.

Considerando los tratamientos establecidos de primera y segunda línea para la población pediátrica, establecidos en las guías ASH mencionadas previamente:
- Se recomienda como primera línea los agentes trombopoyéticos cuando el paciente no cuenta con diagnóstico confirmado de COVID-19.

- La IgIV no es un inmunosupresor y eleva el recuento de plaquetas rápidamente. Sin embargo, no contiene anticuerpos contra el SARS-CoV-2, no mantiene el recuento de plaquetas durante un tiempo prolongado (generalmente < 3-4 semanas) y requiere una visita a un centro de infusión, por lo que se recomienda solo en los casos donde se requiera elevación rápida del conteo de plaquetas por manifestación hemorrágica. El riesgo de infecciones virales trasmitidas por la administración de IgIV se considera extremadamente bajo.

- Si bien no hay literatura sobre infección por SARS-CoV-2 y pacientes sometidos a inmunosupresores, el riesgo de inmunosupresión con dosis altas de corticosteroides, rituximab u otros agentes inmunosupresor es comparable a otros virus.

- Evitar los agentes anti-CD20, ya que deterioran las respuestas humorales a las infecciones de novo y las vacunas.

- No hay datos sobre transfusiones de plaquetas en pacientes con COVID-19, por lo que sus riesgos y beneficios permanecen indefinidos en este entorno específico. Se recomienda trasfusión solo en caso de hemorragias que pongan en riesgo la vida.

En TIP persistente o crónica no se necesita modificación de la terapia establecida para los pacientes estables con dosis bajas de esteroides o inmunosupresores, cambiar los tratamientos requiere una mayor monitorización y podría dar lugar a una recaída, por lo que puede ser más riesgoso que continuar con el statu quo. Para los pacientes con dosis más altas de corticosteroides o medicamentos inmunosupresores, el uso de agentes trombopoyéticos y/o IgIV podría permitir la disminución gradual y posiblemente la interrupción de estos medicamentos.

El tratamiento de un paciente esplenectomizado febril no cambia en el contexto de la COVID-19, y la administración urgente de antibióticos por vía intravenosa es obligatoria hasta que los cultivos bacterianos se documenten como negativos, incluso si la fiebre se atribuye a una infección comprobada o sospechada de SARS-CoV-2.

Aunque los niños son menos susceptibles que los adultos a infección por SARS-CoV-2, es indispensable permanecer vigilantes y cautelosos. Para evitar la 
propagación de la infección, los niños con TIP deben seguir buenas prácticas de higiene: lavado de manos regular, evitar tocarse la boca, la nariz o los ojos, lavado intenso de manos después de regresar de lugares públicos, cubrir boca y nariz al toser o estornudar, estornudos, evitar lugares concurridos y contacto con personas enfermas, en particular aquellas con síntomas respiratorios y fiebre, y encontrar estrategias alternativas para reducir el impacto en la calidad de vida relacionada con la salud.

Al carecer de información específica para este grupo de edad, se consideran para los pacientes pediátricos las mismas recomendaciones que las establecidas para los adultos con TIP en las guías del Reino Unido, aunado a las recomendaciones del consenso de expertos de la ASH sobre el tema, publicados en su portal oficial.

\section{Puntos clave y recomendaciones}

- En la mayoría de los niños el curso de la COVID-19 es asintomático o leve, pudiendo incluso considerarse portadores asintomáticos.

- Se recomienda como primera línea a los agentes trombopoyéticos cuando el paciente no cuenta con diagnóstico confirmado de COVID-19.

- El tratamiento con esteroides debe limitarse, por conllevar riesgo teórico de aumentar la susceptibilidad a la infección; hasta la fecha se desconoce su impacto en el resultado de COVID-19.

- Si se consideran los esteroides deberán administrarse a dosis bajas mínimas necesarias y en ciclos a corto plazo (1-5 días).

- El riesgo de enfermedad severa cuando existe inmunocompromiso es desconocido, por lo que se recomienda evitar inmunosupresores en pacientes de reciente diagnóstico.

- En pacientes con TIP crónica mantener el tratamiento establecido, aunque sean corticosteroides o inmunosupresores, a dosis bajas mínimas requeridas.

- Se recomienda trasfusión de concentrados plaquetarios solo en caso de hemorragias que pongan en riesgo la vida.

- En pacientes postesplenectomizados, cumplir rigurosamente esquemas de antibioticoterapia profiláctica y esquema de vacunación.

- Se sugiere disminuir la frecuencia del recuento de plaquetas y evitar las visitas a los centros de salud.

\section{Conclusiones}

Hasta el momento no parece haber una mayor incidencia de infección o enfermedad grave por COVID-19 en pacientes con TIP, pero aún no existe una recopilación de datos sólida sobre esta cuestión. Si un paciente con TPI conocida y COVID-19 activa muestra una disminución en el recuento de plaquetas a niveles peligrosos (p. ej., < 10-20,000/ul), se debe administrar IgIV para un rápido ascenso plaquetario, y la transfusión de plaquetas reservarse solo para hemorragias graves. Si el paciente ya está tomando un agente trombopoyético, la dosis podría aumentarse o comenzarse una segunda, por ejemplo, agregar romiplostim al eltrombopag o avatrombopag, o agregar eltrombopag o avatrombopag al romiplostim. Se podría considerar que los esteroides a corto plazo (1-5 días) aumentan el recuento de plaquetas, pero se desconoce su impacto en el resultado de COVID-19.

En los pacientes pediátricos será siempre importante estudio de panel viral como etiología de TIP. En esta pandemia será importante realizar estudios epidemiológicos dirigidos a aclarar una posible correlación, pero también para un aviso del diagnóstico de COVID-19 (en particular para pacientes levemente sintomáticos y en niños).

Actualmente no podemos definir si en el futuro se establecerá una asociación entre TIP y COVID-19, así como si el resultado de la TIP será influenciado en términos de cronicidad y respuesta a las terapias TIP. En estos días, cuando cada actividad diaria se ha ralentizado para preservar la salud global, continuar protegiendo el bienestar psicofísico de los pacientes afectados por la TIP permitirá construir su futuro de la mejor manera.

\section{Conflicto de intereses}

Los autores manifiestan no tener conflicto de intereses alguno.

\section{Financiamiento}

La presente investigación no ha recibido ninguna beca específica de agencias de los sectores público, comercial o sin ánimo de lucro.

\section{Responsabilidades éticas}

Protección de personas y animales. Los autores declaran que para esta investigación no se han 
realizado experimentos en seres humanos ni en animales.

Confidencialidad de los datos. Los autores declaran que han seguido los protocolos de su centro de trabajo sobre la publicación de datos de pacientes.

Derecho a la privacidad y consentimiento infor-

mado. Los autores declaran que en este artículo no aparecen datos de pacientes.

\section{Bibliografía}

1. Cines DB, Bussel JD, Liebman HA, Luning Prak ET. The ITP syndrome: pathogenic and clinical diversity. Blood. 2009;113:6511-21.

2. Rodeghiero F, Stasi R, Gernsheimer T, Michel M, Provan D, Arnold DM, et al. Standardization of terminology, definitions and outcome criteria in immune thrombocytopenic purpura in adults and children: report from an international working group. Blood. 2009:113:2386-93.

3. Provan D, Stasi R, Newland AC, Blanchette VS, Bolton-Maggs P, Bussel $\mathrm{JB}$, et al. International consensus report on the investigation and management of primary immune thrombocytopenia. Blood. 2010;115:168-86.

4. Salib M, Clayden R, Clare R, Wang G, Warkentin TE, Crowther MA, et al Difficulties in establishing the diagnosis of immune thrombocytopenia: An agreement study. Am J Hematol. 2016;91:E327-E329.

5. Neunert C, Terrell DR, Arnold DM, Buchanan G, Cines DB, Cooper N, et al. American Society of Hematology 2019 guidelines for immune thrombocytopenia. Blood Adv. 2019;3:3829-66.

6. Sood R, Wong W, Gotlib J, Jeng M, Zehnder JL. Gene expressions and pathway analysis of immune thrombocytopenia purpura. Br J Haematol. 2008;140:99-103.

7. Audia S, Mahévas M, Samson M, Godeau B, Bonnotte B. Pathogenesis of immune thrombocytopenia. Autoimmun Rev. 2017:16(6):620-32.

8. Swinkels M, Rijkers M, Voorberg J, Vidarsson G, Leebeek FWG Jansen AJG. Emerging concepts in immune thrombocytopenia. Front Immunol. 2018:9:880.

9. Li J, Sullivan JA, Ni H. Pathophysiology of immune thrombocytopenia. Curr Opin Hematol. 2018;25:373-81.

10. Gutiérrez-Espíndola G, Martínez-Murillo C, Oropeza-Martínez P. Modelo día práctica clínica en pacientes con púrpura trombocitopénica idiopática. Gac Méd Méx. 2009;145(Supl 1):111-16.

11. Neunert $C$, Cooper N. Evidence-based management of immune thrombocytopenia: ASH guideline update. Hematology Am Soc Hematol Educ Program. 2018;2018(1):568-75.

12. Matzdorff A, Meyer O, Ostermann H, Kiefel V, Eberl W, Kühne T,et al Immune thrombocytopenia - Current diagnostics and therapy: Recommendations of a Joint Working Group of DGHO, OGHO, SGH, GPOH and DGTI. Oncol Res Treat. 2018;41(suppl 5):1-30.

13. Pavord S, Thachil J, Hunt BJ, Murphy M, Lowe G, Laffan M, et al. Practical guidance for the management of adults with immune thrombocytopenia during the COVID-19 pandemic. Br J Haematol. 2020;189:1038-43.

14. Busell J, Cines DB, Cooper N, Dunbar C, Michel M, Rodeghiero F. COVID-19 and ITP: Frequently Asked Questions [Internet]. American Society of Hematology; version 3.0 [última actualización: 15/06/2020]. Disponible en: https://www.hematology.org/covid-19/covid-19-and-itp

15. Guan W, Ni Z, Hu Y, Liang WH, Ou CQ, He JX, et al. Clinical characteristics of coronavirus disease 2019 in China. N Engl J Med. 2020;382:1708-20.

16. Huang C, Wang Y, Li X, Ren L, Zhao J, Hu Y, Zhang L, et al. Clinical features of patients infected with 2019 novel coronavirus in Wuhan, China. Lancet. 2020;395:497-506.

17. Lippi G, Plebani M, Henry BM. Thrombocytopenia is associated with severe coronavirus disease 2019 (COVID-19) infections: A meta-analysis. Clin Chim Acta. 2020;506:145-8.
18. Yang $X$, Yang $Q$, Wang $Y$, Wu $Y, X u$ J, Yu Y, et al. Thrombocytopenia and its association with mortality in patients with COVIDa19. J Thromb Haemost. 2020;18:1469-72.

19. Bomhof G, Mutsaers PGNJ, Leebeek FWG, Te Boekhorst PAW, Hofland J, Croles FN, et al. COVID-19-associated immune thrombocytopenia. Br J Haematol. 2020;190(2):e61-e64.

20. Murt A, Eskazan AE, Yılmaz U, Ozkan T, Ar MC. COVID-19 presenting with immune thrombocytopenia: a case report and review of the literature. J Med Virol. 2021;93(1):43-5.

21. Hu Z, Chen W, Liang W, Xu C, Sun W, Yi Y. Severe exacerbation of immune thrombocytopenia and COVID-19: the favorable response to corticosteroid-based therapy-a case report. Ann Hematol. 2020 Jun 4:13. doi: 10.1007/s00277-020-04070-x. Online ahead of print.

22. Merli M, Ageno W, Sessa F, Salvini M, Caramazza D, Mora B, et al. Recurrence of immune thrombocytopenia at the time of SARS-CoV-2 infection. Ann Hematol. 2020;99(8):1951-2.

23. Zulfiqar $\mathrm{AA}$, Lorenzo-Villalba $\mathrm{N}$, Hassler $\mathrm{P}$, Andrès $\mathrm{E}$. Immune thrombocytopenic purpura in a patient with COVID-19. N Engl J Med. 2020;382(18):e43.

24. World Health Organization. Clinical management of severe acute respiratory infection (SARI) when COVID-19 disease is suspected: interim guidance 13 March 2020 [Internet]. World Health Organization; 13/03/2020. Disponible en: https://apps.who.int/iris/handle/10665/331446

25. Arabi YM, Mandourah Y, Al-Hameed F, Sindi AA, Almekhlafi GA, Hussein MA, et al. Costicosteroid therapy for critical ill patients with Middle East respiratory syndrome. Am J Respir Crit Care Med. 2018;197:757-67.

26. Garabet L, Ghanima W, Hellum M, Sandset PM, Bussel JB, Tran H, et al. Increased microvesicle-associated thrombin generation in patients with immune thrombocytopenia after initiation of thrombopoietin receptor agonists. Platelets. 2020;31:322-8.

27. Doobaree IU, Nandigam R, Bennet D, Newland A, Provan D.Thromboembolism in primary immune thrombocytopenia: a systematic literature review and meta-analysis. Eur J Haematol. 2016:97:321-30.

28. Barnes GD, Burnett A, Allen A, Blumenstein M, Clark NP, Cuker A, et al. Thromboembolism and anticoagulant therapy during the COVID-19 pandemic: interim clinical guidance from the anticoagulation forum. J Thromb Thrombolysis. 2020;50:72-81.

29. Matzdroff A, Beer JH. Immune thrombocytopenia patients requiring anticoagulation--Maneuvering Between Scylla and Charybdis. Semin Hematol. 2013;50:S83-S88.

30. Pishko AM, Misgav M, Cuker A, Cines DB, George JN, Vesely SK, et al. Management of antithrombotic therapy in adults with immune thrombocytopenia (ITP): a survey of ITP specialists and general hematologist-oncologists. J Thromb Thrombolysis. 2018;46:24-30.

31. Elalfy MS, Nugent D. Viruses, anti-viral therapy, and viral vaccines in children with immune thrombocytopenia. Semin Hematol. 2016;53:S70-S72.

32. Cecinati V, Principi N, Brescia L, Giordano P, Esposito S. Vaccine administration and the development of immune thrombocytopenic purpura in children. Hum Vaccin Immunother. 2013;9:1158-62.

33. She J, Liu L, Liu W. COVID-19 epidemic: Disease characteristics in children. J Med Virol. 2020;92:747-54.

34. Dong $Y$, Mo X, Hu Y, Qi X, Jiang F, Jiang Z, Tong S. Epidemiology of COVID-19 among children in China. Pediatrics. 2020;145(6):e20200702.

35. Zimmermann $P$, Curtis $N$. Coronavirus infections in children including COVID-19: An overview of the epidemiology, clinical features, diagnosis, treatment and prevention options in children. Pediatr Infect Dis J. 2020:39:355-68.

36. Ludvigsson JF. Systematic review of COVID-19 in children shows milder cases and a better prognosis than adults. Acta Paediatr. 2020;109:1088-95.

37. Lassandro G, Palladino V, Palmieri VV, Amoruso A, Del Vecchio GC, Giordano P. COVID-19 and children with Immune Thrombocytopenia: emerging issues. Mediterr J Hematol Infect Dis. 2020;12(1):e2020028.

38. Ogimi C, Englund JA, Bradford MC, Qin X, Boeckh M, Waghmare A. Characteristics and outcomes of coronavirus infection in children: The role of viral factors and an immunocompromised state. J Pediatric Infect Dis Soc. 2019;8:21-8. 